\title{
That Escalated Quickly: Remdesivir's Place in Therapy for COVID-19
}

\author{
Matthew R. Davis (D) - Erin K. McCreary · Jason M. Pogue
}

Received: June 9, 2020 / Published online: July 10, 2020

(C) The Author(s) 2020
Keywords: ACTT-1; Antiviral; Coronavirus disease 2019; COVID-19; Remdesivir; SARS$\mathrm{CoV}-2$

Digital Features To view digital features for this article go to https://doi.org/10.6084/m9.figshare.12563738.

M. R. Davis $(\square)$

Department of Clinical Pharmacy, University of California Los Angeles Ronald Reagan Medical Center, Los Angeles, CA, USA

e-mail: MRDavis@mednet.ucla.edu

E. K. McCreary

Division of Infectious Diseases, Department of Medicine, University of Pittsburgh Medical Center, Pittsburgh, PA, USA

J. M. Pogue

Department of Clinical Pharmacy, University of

Michigan College of Pharmacy, Ann Arbor, MI, USA

\section{Key Summary Points}

Results have been mixed for remdesivir in studies of COVID-19, but it is the first effective therapy for COVID-19 regarding shortening time to recovery.

Limited availability of remdesivir requires clinicians to face difficult decisions when prioritizing patients.

This report summarizes and aggregates the available clinical data to aid in clinical decision-making for remdesivir therapy.

Remdesivir is generally well tolerated with comparable rates of adverse events to placebo.

\section{BACKGROUND}

Remdesivir is a nucleoside analog prodrug with potent in vitro activity against numerous human and zoonotic coronaviruses; it has also demonstrated clinical benefit in animal models of SARS-CoV, MERS-CoV, and SARS-CoV-2 infection [1-11]. Antiviral activity is mediated by preferential incorporation of the molecule by viral RNA-dependent RNA polymerase into the 
RNA transcript, leading to delayed chain termination due to steric impedance $[12,13]$. For a detailed discussion of pre-clinical, pharmacology, and pharmacokinetic data, the reader is directed to a recent review by Jorgensen et al. [9]. Since the publication of this review, results from two randomized trials evaluating remdesivir for hospitalized patients with COVID-19 were published. Herein, we aim to aid clinicians in delineating the role of remdesivir for treatment of COVID-19 by critically analyzing and aggregating available, pertinent clinical data from January 1, 2020, to May 31, 2020. Patients represented by these data received remdesivir via a compassionate use pathway or enrollment in a clinical trial. Currently, there is no FDAapproved medication for the treatment of COVID-19. This article is based on previously conducted studies and does not contain any studies with human participants or animals performed by any of the authors.

\section{COMPASSIONATE USE}

Starting on January 25, 2020, clinicians were able to request remdesivir via a compassionate use program for any hospitalized patient with severe COVID-19, defined as either an oxygen saturation of $\leq 94 \%$ breathing ambient air or any oxygen requirement. The first published report of a patient with COVID-19 treated via this program describes a 35-year-old male in Snohomish County, Washington, who initially presented with mild disease but developed bilateral pneumonia requiring $2 \mathrm{~L}$ supplemental oxygen on symptom day 10 [14]. Remdesivir was initiated on symptom day 11 with improvement in clinical status the following day. Two months later, Grein and colleagues reported the safety and efficacy of compassionate use remdesivir in a case series of 61 patients from the US, Europe, Canada, and Japan [15]. The series population represented all patients in the program who received their first dose of remdesivir on or before March 7, 2020, and had clinical data available for at least 1 subsequent day. Notably, eight patients were excluded from the report for lack of post-baseline data or erroneous drug start date, leaving 53 evaluable patients. The majority (75\%) of patients were men with a median age of 64 years (interquartile range [IQR] 48-71) and symptom duration of 12 days (IQR 9-15) prior to remdesivir initiation. The program allocated a 10-day treatment course of remdesivir, which was completed by 40 (75\%) of the population; 3 (6\%) patients did not complete therapy because of discharge from the hospital. The cohort was critically ill at the time of remdesivir initiation with $64 \%$ of patients receiving mechanical ventilation or extracorporeal membrane oxygenation (ECMO), 4\% non-invasive positivepressure ventilation, 9\% high-flow oxygen, 19\% low-flow oxygen, and $4 \%$ ambient air. Patients were followed for median 18 days (IQR 13-23) with $68 \%$ demonstrating improvement in oxygen status and $15 \%$ worsening, including seven $(13 \%)$ deaths. Clinical improvement was defined as a decrease $\geq 2$ points on a 6-point ordinal scale $(1=$ discharge; $2=$ ambient air; $3=$ low-flow oxygen; $4=$ non-invasive oxygen support; $5=$ mechanical ventilation/ECMO; $6=$ death) or discharge. Clinical improvement was most pronounced in patients requiring lowflow or no oxygen $(12 / 12,100 \%)$ compared to $5 / 7(71 \%)$ in those receiving noninvasive ventilation and 16/34 (47\%) in mechanically ventilated patients. While the authors report a cumulative incidence of improvement of $84 \%$ by 28-day follow-up, this was overstated considering deaths were censored events rather than deemed "not improved" [16]. Indeed, when clinical improvement was reanalyzed by the authors using a competing risks approach, cumulative incidence was $74 \%$ [17]. Without a control group, it is difficult to interpret or draw any meaningful conclusions from this data set. On April 30, 2020 [18], Gilead reported > 2000 patients have been treated with remdesivir via compassionate use and expanded access programs; the clinical outcomes of these patients are not currently available.

\section{RANDOMIZED CLINICAL TRIALS}

Wang and colleagues reported results of the first randomized, double-blind, placebo-controlled trial of remdesivir in adult patients with severe 
COVID-19 on April 29, 2020 [19]. Two hundred thirty-six patients from ten hospitals in Hubei, China, were randomized 2:1 (stratified by baseline respiratory status) to receive 10 days of remdesivir $(N=158)$ or placebo $(N=78)$. Due to the local control of the SARS-CoV-2 outbreak, enrollment was terminated prior to the recruitment target of 453 patients, reducing statistical power from 80 to $58 \%$.

The primary outcome was time to clinical improvement within 28 days of randomization. Clinical improvement was defined as a twopoint reduction on a six-point ordinal scale (identical to the scale used in the aforementioned compassionate use case series) or alive discharge (including patients still admitted but meeting discharge criteria for $\geq 72 \mathrm{~h}$ : afebrile, respiratory rate $<24$ breaths per minute, $\mathrm{SpO}_{2}$ $\geq 94 \%$ on room air, resolution of cough). Patients were assessed clinically once daily, and viral RNA samples were collected throughout the study. Use of repurposed medications with in vitro activity against SARS-CoV-2 and other adjunctive therapies was permitted and did not differ between study groups; $28 \%$ of the total population received lopinavir-ritonavir, 32\% interferon alfa-2b, and $66 \%$ corticosteroids. Baseline characteristics were similar between groups. Eighty-two percent of the study population was receiving low-flow supplemental oxygen at the time of enrollment, $15.6 \%$ required high-flow oxygen (18\% vs. $12 \%)$, and only one patient was mechanically ventilated. The median time from symptom onset to enrollment was 10 days (IQR 9-12) for the cohort with no significant difference between groups.

In the intention-to-treat population, no difference was observed in time to clinical improvement in the remdesivir group compared to patients receiving placebo (median 21 days [IQR 13-28] vs. 23 days [IQR 15-28], HR 1.23 [95\% CI 0.87-1.75]). In a post hoc analysis of the subgroup enrolled within 10 days of symptom onset, median time to improvement was 18 days (IQR 12-28) in remdesivir-treated patients compared to 23 days (IQR 15-28) in those receiving placebo (HR $1.52 \quad[95 \%$ CI $0.95-2.42])$. Deaths by day 14 (10\% vs. $9 \%)$ and 28 (14\% vs. $13 \%)$ were similar between groups.
There was also no difference in discharges by day 14 (25\% vs. $23 \%$, remdesivir vs. placebo, respectively). Remdesivir did not impact time to clinical deterioration (HR $0.95 \quad[95 \%$ CI 0.55-1.64]) or quantitative viral load, regardless of time of enrollment. Taken together, these data do not support a clinically meaningful benefit of remdesivir in patients with severe COVID-19, but the potential effect of symptom duration on efficacy warranted further exploration.

The first stage of the National Institute of Allergy and Infectious Diseases (NIAID)-sponsored Adaptive COVID-19 Treatment Trial (ACTT-1) was a randomized, double-blind, placebo-controlled trial assessing the efficacy of a 10-day course of remdesivir for hospitalized patients with severe COVID-19. The trial was conducted at 60 sites and 14 subsites internationally in North America, Europe, and Asia. Patients were randomized in a one-to-one fashion to receive either remdesivir or placebo; randomization was stratified by disease severity and site of enrollment.

The primary outcome was initially difference in clinical status, as defined by an eight-point ordinal scale at day 15 post-enrollment (scale: $1=$ not hospitalized, no limitations of activities; $2=$ not hospitalized, limitation of activities, home oxygen requirement, or both; $3=$ hospitalized, not requiring supplemental oxygen, and no longer requiring ongoing medical care; $4=$ hospitalized, not requiring supplemental oxygen but requiring ongoing medical care; $5=$ hospitalized, requiring any supplemental oxygen; 6 = hospitalized, requiring noninvasive ventilation or high-flow oxygen devices; $7=$ hospitalized, receiving invasive mechanical ventilation or ECMO; 8 = death). However, due to an evolving understanding of the protracted nature of COVID-19 disease progression, trial statisticians blinded to study data proposed a change in the primary outcome to time to recovery, defined as the first day a patient satisfies ordinal scale categories 1,2 , or 3 . Of the 1107 patients assessed for eligibility, 1063 underwent randomization and 1049 received their assigned intervention (531 remdesivir, 518 placebo). As of April 28, 2020, 301 patients (132 remdesivir, 169 placebo) were still enrolled in 
the trial and had not yet achieved the primary outcome or death; therefore, the published data are a preliminary report. However, the trial had completed enrollment at the time of the Data Safety Monitoring Board review, prior to this publication.

Baseline characteristics were well matched between remdesivir and placebo arms with a mean age of 58.9 years. Sixty-five percent of patients were male. The most common comorbidities present were hypertension (49.6\%), obesity (37.0\%), and diabetes (29.7\%). Median time from symptom onset to randomization was 9 days (IQR 6-12 days) for remdesivir and 9 days (IQR 7-13 days) for placebo. The most common baseline ordinal category score was 5 (hospitalized, requiring supplemental oxygen), which included 222 patients $(41.0 \%)$ for remdesivir and 199 patients (38.1\%) for placebo. This was followed by ordinal category 7 $(23.1 \%$ vs. $28.2 \%)$, category $6(18.1 \%$ vs. $19.0 \%)$, and category 4 (12.4\% vs. $11.5 \%)$.

Patients receiving remdesivir had a statistically significant shorter time to recovery than those on placebo (median 11 days vs. 15 days; rate ratio [RR] for recovery $1.32 ; 95 \%$ confidence interval [CI], 1.12-1.55; $P<0.001$ ). The benefit in time to recovery was primarily driven by patients in ordinal category 5 (low-flow supplemental oxygen) at baseline (RR 1.47; 95\% CI 1.17-1.84). Remdesivir was also superior to placebo in the key secondary outcome assessing the odds of improvement in the ordinal scale on day 15 via a proportional odds model (odds ratio $1.50 ; 95 \%$ CI $1.18-1.91 ; P=0.0001)$. A test of interaction between treatment arm and baseline ordinal category was not significant, and an analysis of the primary outcome adjusted for baseline ordinal score provided a similar treatment-effect estimate (RR 1.31; 95\% CI 1.12-1.54; 1017 patients). Unlike other reports $[19,20]$, no discernible effect was evident in the pre-specified subgroup analysis of symptom duration prior to randomization [patients experiencing symptoms $\leq 10$ days prior to randomization (RR for recovery 1.28; 95\% CI $1.05-1.57$ ) vs. $>10$ days (RR 1.38; 95\% CI 1.05-1.81)]. This finding refutes the use of arbitrary symptom duration cutoffs (i.e., $<10$ days) for treatment allocation, which is unsupported by the sum total of the evidence.

A hazard ratio showed a numerical decrease in mortality with remdesivir $(0.70 ; 95 \%$ CI 0.47-1.04; 1059 patients). Kaplan-Meier estimates of mortality by 14 days were $7.1 \%$ and $11.9 \%$ in the remdesivir and placebo arms, respectively, but day 28 mortality estimates could not be performed because of the significant proportion of patients that had yet to complete final follow-up visits. This reduction in mortality was most apparent for patients in category 5 at baseline, with Kaplan-Meier estimates of $2.4 \%$ vs. $10.9 \%$ (HR 0.22 [95\% CI $0.02-0.58]$ ). A similar reduction was not seen for category 7 (11.3\% vs. $14.1 \%$; HR 1.06 [95\% CI $0.59-1.92])$ or category $6(15.2 \%$ vs. $14.7 \%$; HR 1.12 [95\% CI 0.52-2.38]). Remdesivir also demonstrated greatest benefit in potentially preventing clinical deterioration for patients in category 5 . Of patients with both baseline and day 15 ordinal scores available, patients who worsened in each category were numerically lower for remdesivir versus placebo: category 4 (8.3\% vs. $15.7 \%)$, category 5 ( $8.7 \%$ vs. $21.8 \%)$, category $6(25.4 \%$ vs. $33.7 \%)$, and category 7 (13.9\% vs. $16.5 \%)$.

In this preliminary report of the ACTT-1 data, remdesivir reduced time to recovery in the overall study population by 4 days. The greatest benefit appeared to be in patients requiring lowflow supplemental oxygen at baseline; however, analysis of recovery in patients with more severe baseline status demonstrated wide confidence intervals, which were not adjusted for multiplicity. Final data are not yet available for the full cohort, which complicates interpretation of results in patients with more severe presentation of disease that often have more protracted courses of illness [21-23]. Generalizability outside of the US might be limited considering this comprised $80 \%$ of the enrolled patients. Additionally, following the initial announcement of these results on April 29, 2020 [24], the decision was made to unblind the study and offer remdesivir to the remaining 169 patients receiving placebo who had not completed the day 29 follow-up visit. It is unknown how many placebo-randomized patients have received remdesivir at this point, or how future 
results generated from these data will be confounded because of the inextricable biases associated with open-label treatment decisions.

More recently, Goldman and colleagues presented the results of a randomized, open-label, multicenter, international trial comparing a 5- or 10-day course of remdesivir for patients with COVID-19 and an oxygen saturation $\leq$ $94 \%$ on ambient air or requiring supplemental oxygen (SIMPLE-1, Gilead-sponsored trial) [20]. Exclusion criteria included baseline need for mechanical ventilation and/or ECMO, multiorgan dysfunction, estimated creatinine clearance $<50 \mathrm{ml} / \mathrm{min}$ (notably more stringent than the previous two trials), aspartate aminotransferase or alanine aminotransferase five times the upper limit of normal, and concurrent therapy with putative SARS-CoV-2 activity within $24 \mathrm{~h}$. The primary end point was clinical status assessed at day 14 on a 7-point ordinal scale ( $1=$ death; $2=$ hospitalized, receiving invasive mechanical ventilation or ECMO; 3 = hospitalized, receiving noninvasive ventilation or highflow oxygen devices; $4=$ hospitalized, requiring low-flow supplemental oxygen; $5=$ hospitalized, not requiring supplemental oxygen but receiving ongoing medical care; $6=$ hospitalized, requiring neither supplemental oxygen nor ongoing medical care; $7=$ not hospitalized). Readers should note this is the inverse order of outcomes in relation to that presented in ACTT1 (i.e., death is 8 in ACTT-1). Secondary outcomes included time to clinical improvement (a 2 -point change from baseline on the ordinal scale), time to recovery (defined as a 6 or 7 on this scale, which is analogous to the primary outcome in ACTT-1), death, and adverse events. The sample size was selected to have $85 \%$ power to demonstrate superiority of a 10-day course at an odds ratio for improvement of 1.75 .

Three hundred ninety-seven patients were included in this study including 200 randomized to 5 days of therapy and 197 to 10 days. Baseline characteristics were mostly similar between the groups. The median age of the cohort was 61 years (50-71), 64\% were male, and $70 \%$ were white. Common comorbidities included hypertension (50\%) and diabetes (23\%). The median duration of symptoms prior to receipt of remdesivir was 8 (IQR 5-11) and 9
(IQR 6-12) days in the 5- and 10-day arm, respectively. There were more patients in the 10-day group who started at a higher severity of illness on the ordinal scale (5\% vs. $2 \%$ were on mechanical ventilation or ECMO [despite these patients being excluded from enrollment] and $30 \%$ vs. $24 \%$ were receiving high-flow or noninvasive mechanical ventilation at baseline, respectively). These differences in baseline clinical status between arms were statistically significant when analyzed using the Wilcoxonrank sum test $(P=0.02)$. Patients were not required to complete the entire course of therapy if they were otherwise ready to be discharged. Sixteen (8\%) patients in the 5-day arm were discharged prior to completing the full course whereas 68 (35\%) patients in the 10-day arm were discharged home prior to treatment completion. Overall, 86 (44\%) patients in the 10-day arm completed the full course of treatment with a median duration of 9 days (IQR 5-10).

After adjustment for differences in baseline clinical status between the two groups, receipt of a 10-day course of remdesivir was not associated with an improvement in clinical status at day 14 on the 7 -point ordinal scale $(P=0.14)$. Similarly, receipt of 10 days of remdesivir was not associated with an improvement in time to clinical improvement [median 11 vs. 10 days, adjusted OR 0.79 (0.61-1.01), time to recovery (median 11 vs. 10 days, adjusted OR 0.81 $(0.64-1.04)$, or death $(11 \%$ vs. $8 \%, p=0.14)$ ]. The authors concluded there is no significant difference between a 5- and 10-day treatment course of remdesivir.

In a post hoc analysis, the investigators sought to determine whether any subpopulation would benefit from days 6 to 10 of therapy. To accomplish this, the investigators assessed oxygen status at day 5 to create a new "baseline" and evaluated oxygen status at day 14 as a function of whether or not the patients got 5 additional days of therapy (i.e., the 10-day group versus the 5-day group). For patients on ambient air, low-flow oxygen, or high-flow oxygen at day 5 , there was no association between receipt of an additional 5 days of therapy and day 14 outcome. Numerically more patients who received 10 days of therapy in the 
high-flow oxygen group progressed to mechanical ventilation or death compared to those treated for 5 days ( $41 \%$ vs. $25 \%$, respectively). For patients receiving invasive mechanical ventilation on day 5 , patients receiving 10 days of therapy appeared to have a lower incidence of day 14 mortality [7/41 (17\%) vs. $10 / 25(40 \%)$ ]. This post hoc analysis should be interpreted with caution considering patients were not randomized to receive additional therapy based on day 5 clinical status; rather this randomization was performed 5 days prior, leading to the potential for imbalances in patient characteristics between arms in these small subgroups. Additionally, the inconsistency in potential benefit of prolonged therapy between the high-flow oxygen and mechanical ventilation subgroups further emphasizes the need for caution in interpretation.

\section{SAFETY}

Remdesivir appears to be safe and well tolerated. In a combined analysis by the European Medicines Association of four studies comprised of 138 healthy volunteers, adverse events occurring in five or more patients included phlebitis $(n=8)$, constipation $(n=7)$, headache $(n=6)$, ecchymosis $(n=5)$, nausea $(n=5)$, and pain in extremities $(n=5)$. Transient treatment-emergent elevations in alanine aminotransferase (ALT) and aspartate aminotransferase (AST) observed in healthy volunteers were all grade 1 or grade 2 in severity; incidence rates were not reported $[25,26]$.

The clinical data substantiate the safety demonstrated in phase I studies. In a randomized, placebo-controlled trial of patients with severe COVID-19 in China, adverse events occurred in $66 \%$ and $64 \%$ of patients receiving remdesivir and placebo, respectively [19]. The most common adverse events were constipation ( $14 \%$ vs. $15 \%$ ), AST elevation ( $5 \%$ vs. $12 \%$ ), and thrombocytopenia (10\% vs. $6 \%)$. Two remdesivir-treated patients discontinued the medication because of ALT elevation. In SIMPLE-1, serious adverse events were more frequent in patients randomized to 10-day therapy compared to 5 day (35\% vs. $21 \%$ ), which was largely driven by respiratory adverse events including respiratory failure/acute respiratory distress syndrome ( $16.7 \%$ vs. $8 \%)$. Laboratory abnormalities such as grade 3 or 4 decrease in creatinine clearance were numerically higher in patients receiving 10 days of therapy (13\% vs. $9.3 \%)$, but there were minimal differences between arms in ALT $(8.3 \%$ vs. $6.2 \%)$ and AST elevation $(5.8 \%$ vs. $7.2 \%)$. More patients discontinued remdesivir treatment because of adverse events in the 10 -day group compared to 5 days $(10 \%$ vs. $4 \%$, respectively). In the absence of a control group, it is difficult to discern if the higher rates of serious adverse events in the 10-day arm were due to increased remdesivir exposure, sicker patients, or a combination of these factors.

In ACTT-1, rates of serious adverse events were lower in remdesivir-treated patients than in those receiving placebo $(21.1 \%$ vs. $27 \%$, respectively), including lower rates of elevated transaminases in $4.1 \%$ vs. $5.9 \%$ [27]. The most common adverse events observed were anemia (7.9\% vs. 9.0\%), combined acute kidney injury or decreased estimated glomerular filtration rate or creatinine clearance $(7.4 \%$ vs. $7.3 \%)$, pyrexia $(5.0 \%$ vs. $3.3 \%)$, and hyperglycemia $(4.1 \%$ vs. $3.3 \%)$. The data suggest remdesivir has an acceptable tolerability profile, and the high incidence of adverse events in clinical studies for patients with COVID-19 likely is more reflective of disease than remdesivir toxicity. This is supported by the comparable rates of adverse events and serious adverse events across the spectra in placebo-treated patients.

The inclusion of sulfobutylether-beta-cyclodextrin (SBECD) as a solubilizing agent is likely of minimal clinical significance [28, 29], but clinicians are advised to weigh perceived risks and benefits of therapy in patients with renal dysfunction, noting these patients were excluded from clinical trials.

\section{REMDESIVIR'S ROLE IN THERAPY}

Results from clinical trials support remdesivir as the first antiviral therapy for SARS-CoV-2 to have a positive impact on patient outcomes (see Table 1). In response to the preliminary results of ACTT-1, the Food and Drug Administration issued Emergency Use Authorization of remdesivir for 
Table 1 Clinical data comparison

\begin{tabular}{|c|c|c|c|c|}
\hline Study & Population & Interventions & Primary end point & Comments \\
\hline $\begin{array}{l}\text { Wang } \\
\text { et al. } \\
{[19]}\end{array}$ & $\begin{array}{l}\text { Pneumonia on } \\
\text { imaging }+ \\
\mathrm{SpO}_{2} \leq 94 \% \text { OR } \\
\mathrm{PF} \\
\text { ratio } \leq 300 \mathrm{mmHg} \\
+\leq 12 \text { days of } \\
\text { symptom onset }\end{array}$ & $\begin{array}{l}\text { RDV vs. PBO } \\
\text { 10-day course } \\
\text { Allocation } \\
2: 1 \\
\text { ITT: } \\
N=236\end{array}$ & $\begin{array}{l}\text { Time to clinical improvement } \\
\text { within } 28 \text { days (2-point } \\
\text { reduction on 6-point ordinal } \\
\text { scale, discharge or meeting } \\
\text { discharge criteria } \geq 72 \mathrm{~h}^{\mathrm{d}} \text { ) } \\
\text { RDV median } 21 \text { days (IQR } \\
\text { 13-28) vs. PBO median } 23 \text { days } \\
\text { (IQR 15-28) }\end{array}$ & $\begin{array}{l}\text { No benefit with remdesivir in } \\
\text { primary outcome or secondary } \\
\text { outcomes including: } 28 \text {-day } \\
\text { mortality, improvement at } 14 \\
\text { days, viral load reduction, time } \\
\text { to deterioration } \\
\text { Study terminated early because of } \\
\text { outbreak containment }\end{array}$ \\
\hline $\begin{array}{c}\text { Beigel } \\
\text { et al. } \\
{[27]}\end{array}$ & $\begin{array}{l}\text { LRTI by one of: } \\
\text { Pneumonia on } \\
\text { imaging } \\
\mathrm{SpO}_{2} \leq 94 \% \\
\text { Oxygen support } \\
+ \\
\text { Any duration of } \\
\text { illness } \\
+ \\
\text { SARS-CoV- } 2 \\
\text { PCR } \leq 72 \mathrm{~h}^{\mathrm{b}}\end{array}$ & $\begin{array}{l}\text { RDV vs. PBO } \\
\text { 10-day course } \\
\text { Allocation } \\
1: 1 \\
\text { ITT: } \\
\quad N=1059\end{array}$ & $\begin{array}{l}\text { Time to recovery (first day a } \\
\text { patient met category } 1-3^{\mathrm{c}} \text { on } \\
\text { ordinal scale) } \\
\text { RDV median } 11 \text { days vs. } \mathrm{PBO} \\
\text { median } 15 \text { days; rate ratio for } \\
\text { recovery } 1.32 \text { ( } 95 \% \text { CI } 1.12-- \\
1.55 ; P<0.001 ; 1059 \text { patients) }\end{array}$ & $\begin{array}{l}\text { Benefit driven by patients } \\
\text { requiring low-flow } \\
\text { supplemental oxygen at } \\
\text { baseline, but no significant } \\
\text { interaction detected between } \\
\text { baseline status and ordinal } \\
\text { score; numerically lower } \\
\text { mortality in RDV arm ( } 7.1 \% \\
\text { vs. } 11.9 \%) \\
\text { Preliminary report, } 301 \text { patients } \\
\text { continuing trial at time of } \\
\text { publication }\end{array}$ \\
\hline $\begin{array}{c}\text { Goldman } \\
\text { et al. } \\
{[20]}\end{array}$ & $\begin{array}{l}\text { Radiographic } \\
\text { infiltrates } \\
+ \\
\text { Ambient } \\
\text { SpO } 2 \leq 94 \% \text { OR } \\
\text { Supplemental oxygen } \\
\text { MV/ECMO and } \\
\text { multiorgan failure } \\
\text { excluded }\end{array}$ & $\begin{array}{l}\text { RDV } 5 \text { vs. } \\
10 \text { days } \\
\text { Allocation } \\
1: 1 \\
\text { ITT: } \\
N=397\end{array}$ & $\begin{array}{l}\text { Day } 14 \text { clinical status (7-point } \\
\text { ordinal scale) } \\
\text { No significant difference } \\
\quad(P=0.14) \text { by Wilcoxon rank- } \\
\text { sum stratified by baseline } \\
\text { clinical status }\end{array}$ & $\begin{array}{l}\text { Significantly worse baseline } \\
\text { clinical status in } 10 \text {-day group } \\
(P=0.02) \text {; similar outcomes } \\
\text { after adjustment for baseline } \\
\text { clinical status }\end{array}$ \\
\hline
\end{tabular}

$P F$ ratio ratio of arterial oxygen partial pressure to fractional inspired oxygen, $R D V$ remdesivir, $P B O$ placebo, $I T T$ intention to treat, $M V$ mechanical ventilation, ECMO extracorporeal membrane oxygenation

a Oxygen support $=$ supplemental oxygen, mechanical ventilation, or ECMO

b Exceptions permitted for PCR $>72 \mathrm{~h}$ if site unable to obtain a repeat sample

c Category 1 = not hospitalized, no limitations; category $2=$ not hospitalized, limitations; category $3=$ hospitalized, no ongoing medical care

d Discharge criteria $=$ afebrile, respiratory rate $<24$ breaths per minute, $\mathrm{SpO}_{2} \geq 94 \%$ on room air, resolution of cough 
patients with severe COVID-19 on May 1, 2020, and Gilead donated 1.5 million doses of medication to be distributed via this program. With extremely limited supply, clinicians must make difficult decisions about which patients to prioritize to receive remdesivir therapy. To analyze this, we combined day 14 or 15 outcomes in patients from ACTT-1 and SIMPLE-1 (Fig. 1) to further elucidate the potential benefit of remdesivir as a function of baseline oxygen status.

\section{Patients Admitted to the Hospital, but not Requiring Supplemental Oxygen} (Fig. 1a)

In ACTT-1, treatment with remdesivir was associated with an improved ordinal score at day 15 (OR 1.51; 95\% CI 0.76-3.00) compared to placebo in hospitalized patients breathing ambient air. This included an increased number of patients discharged or able to be discharged (90\% vs. $78 \%$ ) and a decreased number of patients with severity of disease progression ( $8 \%$ vs. $16 \%$ ). There was only one death in each arm. Crude outcomes look slightly worse in the small number of patients who meet these criteria in SIMPLE-1 ( $n=52$ for both arms combined) with $25 \%$ of patients demonstrating disease progression; however, these data are generally supportive of the findings from ACTT-1. Additionally, topline data from a placebo-controlled trial (SIMPLE-2) in mildmoderate illness (oxygen saturation $>94 \%$ on room air) support a modest benefit in improvement in day 11 status with receipt of either a 5- or 10-day course of remdesivir [30]. While these data support a small benefit of remdesivir in this patient population in terms of time to clinical improvement, there does not appear to be a difference in progression of severity of disease or death, likely because of the low incidence rate of these end points in mild-moderately ill patients.

\section{Patients Requiring Supplemental Low- Flow Oxygen (Fig. 1b)}

Data demonstrate a clinically meaningful benefit of remdesivir in patients requiring low-flow supplemental oxygen at baseline. In ACTT-1, both a mortality benefit (HR 0.22 [95\% CI
$0.08-0.58]$ ) and decreased time to recovery (RR 1.47 [95\% CI 1.17-1.84]) were demonstrated with remdesivir compared to placebo. Remdesivir outcome data from SIMPLE-1 are consistent with the findings in this subgroup, with similar rates of discharge $(70.3 \%)$, disease progression (13.9\%), and death (3.3\%) to those seen in ACTT1. It is important to acknowledge that remdesivir failed to demonstrate a significant benefit in the small, randomized, placebo-controlled trial by Wang and colleagues where $>80 \%$ of the population fit this baseline category. Whether this was driven by poor overall response in both groups (median time to improvement was nearly twice as long in this trial compared to ACTT-1 and SIMPLE-1), limited sample size, or remdesivir failure remains unclear.

\section{Patients Requiring High-Flow Nasal Cannula or Noninvasive Ventilation (Fig. 1c)}

In ACTT-1, the recovery rate ratio for remdesivir in patients requiring high-flow nasal cannula or noninvasive ventilation was $1.20(0.79-1.81)$ compared to placebo, with a higher proportion of patients being discharged ( $58 \%$ vs. $44 \%)$ and a slightly lower rate of progression ( $25 \%$ vs. $34 \%)$ at day 15. Unfortunately, benefit was not seen in this subgroup in SIMPLE-1. At day 14 , only $32 \%$ of patients were discharged, and, perhaps more alarmingly, $43 \%$ had progressed to either requiring invasive mechanical ventilation or death. Death rates in remdesivir-treated patients in both ACTT-1 and SIMPLE-1 were similar to the placebo group in ACTT-1. Furthermore, in patients in this subgroup on day 5 in the SIMPLE- 1 trial, an additional 5 days of remdesivir did not improve day 14 outcomes compared to patients who stopped therapy at day 5 . Taken together, these data do not support a clinically meaningful benefit of remdesivir in patients requiring high-flow oxygen support on noninvasive ventilation.

\section{Patients Requiring Invasive Mechanical Ventilation (Fig. 1d)}

In both ACTT-1 and SIMPLE-1, remdesivir failed to demonstrate any benefit in patients requiring 

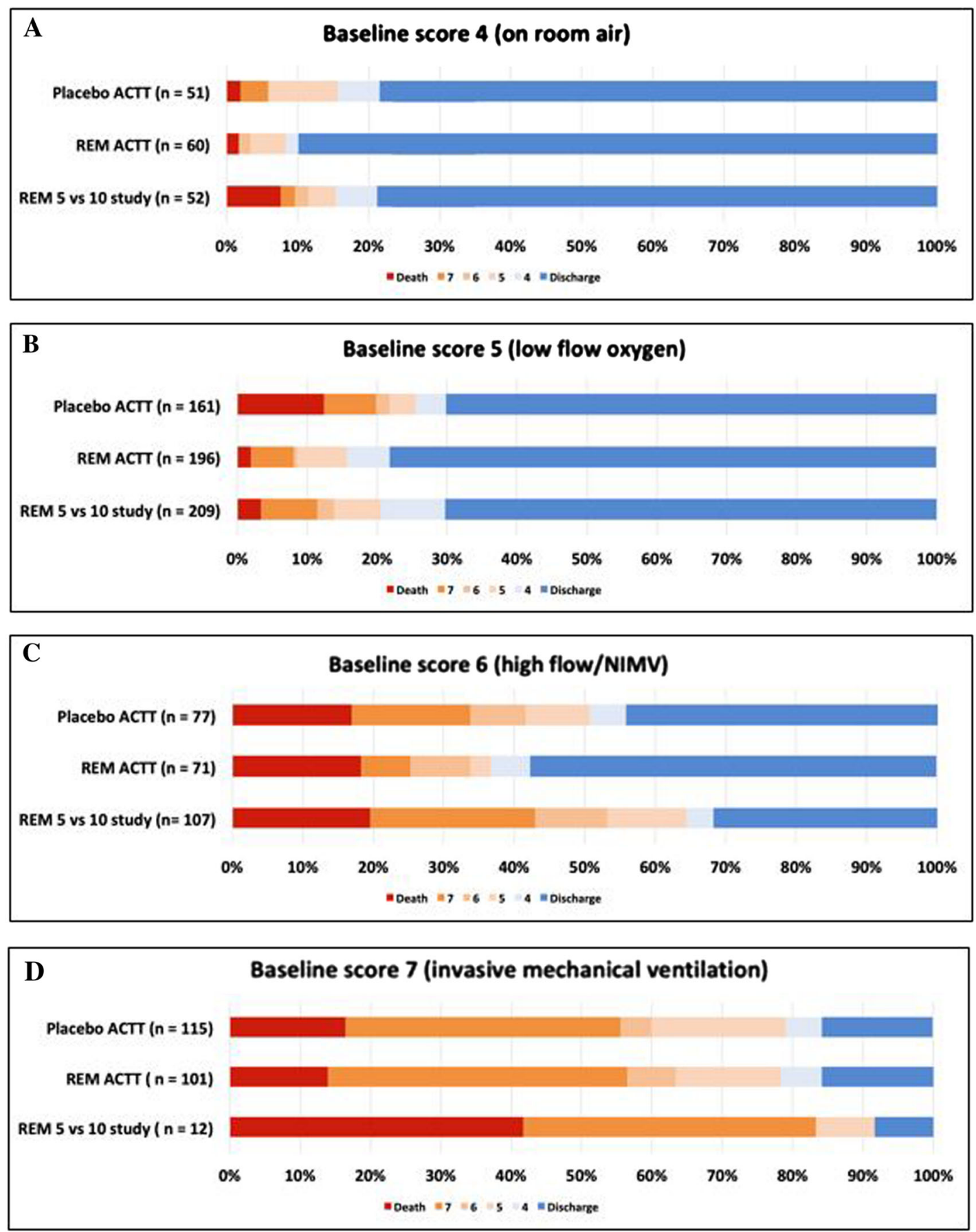

"Certainty of effects cannot be determined, particularly in underrepresented subgroups

Fig. 1 Clinical outcomes on day 14 or 15 by baseline clinical status

mechanical ventilation and/or receiving ECMO. This is particularly notable in ACTT- 1 as this represented the second largest subgroup of patients $(n=272)$ in this trial. Although
SIMPLE-1 suggested a lower mortality rate in patients who were mechanically ventilated patients on day 5 with an additional 5 days of therapy, this finding must be interpreted in the 
context of the results from ACTT-1 where 10 days of remdesivir did not demonstrate any advantage over placebo at day 15 end points. Additionally, the small subset of patients requiring mechanical ventilation and/or receiving ECMO from SIMPLE-1 had higher mortality compared to placebo patients from ACTT-1 (41.7\% vs. $16.5 \%)$ and lower discharge (8.3\% vs. $15.7 \%)$ by day 14 or 15 .

\section{FUTURE STUDIES}

Ongoing international comparative studies will provide additional insights into the role of remdesivir in the treatment of COVID-19. The WHO-sponsored SOLIDARITY trial is currently enrolling in over 400 hospitals in 35 countries with nearly 3500 patients participating to date [31]. The trial seeks to compare local standard of care to standard of care plus remdesivir, lopinavir-ritonavir (LPV/r), hydroxychloroquine, or LPV/r and interferon beta-1a. Additionally, the DisCoVeRy trial sponsored by the French public research organization, Inserm, is also enrolling at 34 registered sites in France and Luxembourg with a target enrollment of 3100 patients [32]. DisCoVeRy is comparing standard of care to standard of care plus remdesivir, LPV/r, hydroxychloroquine, or LPV/r and interferon beta-1a. The second phase of the Adaptive COVID-19 Treatment Trial (ACTT-2) is underway, which aims to characterize the role of remdesivir in combination with baracitinib, an immunomodulatory Janus kinase inhibitor [33]. Finally, the results from SIMPLE-2, which evaluated 5 or 10 days of remdesivir plus standard of care compared to standard of care alone in the treatment of moderate COVID-19, are anticipated to be fully published soon.

In review of the available clinical data, several unanswered questions remain. First and foremost, since the publication of these trials the largest randomized, adaptive clinical trial in the world (RECOVERY) demonstrated a mortality benefit in patients treated with dexamethasone compared to standard of care. While the majority of patients in both groups in the Wang study received corticosteroids, it is unknown how many patients in ACTT-1 or SIMPLE-1 were exposed to corticosteroids during their hospitalization and if this impacted clinical outcomes. The role of remdesivir in combination with other agents, particularly immunomodulators, requires further exploration. Second, while remdesivir has failed to demonstrate a benefit in critically ill patients receiving mechanical ventilation or ECMO at baseline, the full follow-up data from ACTT-1 may provide additional insight into patient populations likely to have prolonged courses of disease. Lastly, data from special populations including immunocompromised patients, pediatrics, and pregnant patients are needed to inform clinical decisions and drug prioritization efforts.

\section{CONCLUSION}

Given the limited availability of remdesivir, we believe treatment should be prioritized for hospitalized patients requiring low-flow supplemental oxygen as it appears these patients derive the most benefit. The data also support some benefit in hospitalized patients breathing ambient air, and if there is adequate drug supply it may be reasonable to treat this patient population. The current data do not suggest benefit for patients who have further progressed in their illness to requiring high-flow oxygen or noninvasive or invasive mechanical ventilation, and alternative therapeutic options should be further assessed in these patients. Notably, while it appears progression of disease plays an important role in the efficacy of remdesivir, the amount of time from onset of symptoms does not. Current evidence does not support a symptom onset cutoff for therapeutic remdesivir decisions; rather, severity of illness should guide these choices. Finally, we recommend a treatment duration of 5 days for all patients based on the summary of the evidence. Overall, remdesivir is a well-tolerated therapeutic agent with demonstrable benefits in the treatment of COVID-19.

\section{ACKNOWLEDGMENTS}

Funding. No funding or sponsorship was received for this study or publication of this article. 
Authorship. All named authors meet the International Committee of Medical Journal Editors (ICMJE) criteria for authorship for this article, take responsibility for the integrity of the work as a whole, and have given their approval for this version to be published.

Disclosures. Matthew R. Davis, Erin K. McCreary, and Jason M. Pogue declare they have no conflicts of interest.

Compliance with Ethics Guidelines. This article is based on previously conducted studies and does not contain any studies with human participants or animals performed by any of the authors.

Open Access. This article is licensed under a Creative Commons Attribution-NonCommercial 4.0 International License, which permits any non-commercial use, sharing, adaptation, distribution and reproduction in any medium or format, as long as you give appropriate credit to the original author(s) and the source, provide a link to the Creative Commons licence, and indicate if changes were made. The images or other third party material in this article are included in the article's Creative Commons licence, unless indicated otherwise in a credit line to the material. If material is not included in the article's Creative Commons licence and your intended use is not permitted by statutory regulation or exceeds the permitted use, you will need to obtain permission directly from the copyright holder. To view a copy of this licence, visit http:// creativecommons.org/licenses/by-nc/4.0/.

\section{REFERENCES}

1. Wang $\mathrm{M}$, Cao R, Zhang L, et al. Remdesivir and chloroquine effectively inhibit the recently emerged novel coronavirus (2019-nCoV) in vitro. Cell Res. 2020;30(3):269-71.

2. Sheahan TP, Sims AC, Leist SR, et al. Comparative therapeutic efficacy of remdesivir and combination lopinavir, ritonavir, and interferon beta against MERS-CoV. Nature communications. 2020;11(1): 222.
3. Lo MK, Jordan R, Arvey A, et al. GS-5734 and its parent nucleoside analog inhibit Filo-, Pneumo-, and Paramyxoviruses. Sci Rep. 2017;7:43395.

4. Brown AJ, Won JJ, Graham RL, et al. Broad spectrum antiviral remdesivir inhibits human endemic and zoonotic deltacoronaviruses with a highly divergent RNA dependent RNA polymerase. Antivir Res. 2019;169:104541.

5. Sheahan TP, Sims AC, Graham RL, et al. Broadspectrum antiviral GS-5734 inhibits both epidemic and zoonotic coronaviruses. Sci Transl Med. 2017. https://doi.org/10.1126/scitranslmed.aal3653.

6. Pruijssers AJ, George AS, Schafer A, et al. Remdesivir potently inhibits SARS-CoV-2 in human lung cells and chimeric SARS-CoV expressing the SARS-CoV-2 RNA polymerase in mice. bioRxiv. 2020. https:// doi.org/10.1101/2020.04.27.064279 (Published Online).

7. Gordon CJ, Tchesnokov EP, Feng JY, Porter DP, Gotte $M$. The antiviral compound remdesivir potently inhibits RNA-dependent RNA polymerase from Middle East respiratory syndrome coronavirus. J Biol Chem. 2020;295(15):4773-9.

8. Gordon CJ, Tchesnokov EP, Woolner E, et al. Remdesivir is a direct-acting antiviral that inhibits RNA-dependent RNA polymerase from severe acute respiratory syndrome coronavirus 2 with high potency. J Biol Chem. 2020;295(20):6785-97.

9. Jorgensen SCJ, Kebriaei R, Dresser LD. Remdesivir: review of pharmacology, pre-clinical data and emerging clinical experience for COVID-19. Pharmacotherapy. 2020. https://doi.org/10.1002/phar. 2429.

10. Agostini ML, Andres EL, Sims AC, et al. Coronavirus susceptibility to the antiviral remdesivir (GS-5734) is mediated by the viral polymerase and the proofreading exoribonuclease. mBio. 2018;9(2):e0022118.

11. Williamson BN, Feldmann F, Schwarz B, et al. Clinical benefit of remdesivir in rhesus macaques infected with SARS-CoV-2. bioRxiv. 2020. https:// doi.org/10.1101/2020.04.15.043166 (Published Online).

12. Eastman RT, Roth JS, Brimacombe KR, et al. Remdesivir: a review of its discovery and development leading to emergency use authorization for treatment of COVID-19. ACS Cent Sci. 2020. https://doi.org/10.1021/acscentsci.0c00489.

13. Siegel D, Hui HC, Doerffler E, et al. Discovery and synthesis of a phosphoramidate prodrug of a Pyrrolo[2,1-f][triazin-4-amino] Adenine C-Nucleoside 
(GS-5734) for the treatment of ebola and emerging viruses. J Med Chem. 2017;60(5):1648-61.

14. Holshue ML, DeBolt C, Lindquist $S$, et al. First case of 2019 novel coronavirus in the United States. N Engl J Med. 2020;382(10):929-36.

15. Grein J, Ohmagari N, Shin D, et al. Compassionate use of remdesivir for patients with severe Covid-19. N Engl J Med. 2020.

16. Hoffmann C. Compassionate use of remdesivir in Covid-19. N Engl J Med. 2020;382(25):e101.

17. Grein J, Myers RP, Brainard D. Compassionate use of remdesivir in Covid-19. Reply. N Engl J Med. 2020;382:2327-36.

18. Gilead Q10. 2020 Earnings Results-April 30, 2020. 2020; http://investors.gilead.com/static-files/ af4599eb-4fb8-4cf7-96a1-38caf477e9b4. Accessed 3 June 2020.

19. Wang Y, Zhang D, Du G, et al. Remdesivir in adults with severe COVID-19: a randomised, double-blind, placebo-controlled, multicentre trial. The Lancet. 2020;395(10236):1569-78.

20. Goldman JD, Lye DCB, Hui DS, et al. Remdesivir for 5 or 10 days in patients with severe Covid-19. $\mathrm{N}$ Engl J Med. 2020. https://doi.org/10.1056/ nejmoa2015301 (Published Online).

21. Bhatraju PK, Ghassemieh BJ, Nichols $M$, et al. Covid-19 in critically ill patients in the Seattle Region -case series. N Engl J Med. 2020;382(21): 2012-22.

22. Wu C, Chen X, Cai Y, et al. Risk factors associated with acute respiratory distress syndrome and death in patients with coronavirus disease 2019 Pneumonia in Wuhan, China. JAMA Intern Med. 2020;180(7):934-943.

23. Guan WJ, Ni ZY, Hu Y, et al. Clinical characteristics of coronavirus disease 2019 in China. N Engl J Med. 2020;382(18):1708-20.

24. NIH Clinical Trial Shows Remdesivir Accelerates Recovery from Advanced COVID-19. News Releases 2020; https://www.niaid.nih.gov/news-events/nihclinical-trial-shows-remdesivir-accelerates-recoveryadvanced-covid-19. Accessed 31 May 2020.
25. Summary on compassionate use. Remdesivir. EMA/ 178637/2020-Rev.1. 03 April 2020. 2020; https:// www.ema.europa.eu/en/documents/other/summa ry-compassionate-use-remdesivirgilead_en.pdf. Accessed 26 May 2020.

26. FACT SHEET FOR HEALTH CARE PROVIDERS EMERGENCY USE AUTHORIZATION (EUA) OF REMDESIVIR (GS-5734 ${ }^{\mathrm{TM}}$ ). 2020; https://www.fda. gov/media/137566/download. Accessed 25 May 2020.

27. Beigel JH, Tomashek KM, Dodd LE, et al. Remdesivir for the treatment of Covid-19-preliminary report. $\mathrm{N}$ Engl J Med. 2020. https://doi.org/10.1056/ NEJMoa2007764.

28. Turner RB, Martello JL, Malhotra A. Worsening renal function in patients with baseline renal impairment treated with intravenous voriconazole: a systematic review. Int $\mathrm{J}$ Antimicrob Agents. 2015;46(4):362-6.

29. Kiser TH, Fish DN, Aquilante CL, et al. Evaluation of sulfobutylether-beta-cyclodextrin (SBECD) accumulation and voriconazole pharmacokinetics in critically ill patients undergoing continuous renal replacement therapy. Crit Care. 2015;19:32.

30. Gilead Announces Results From Phase 3 Trial of Remdesivir in Patients With Moderate COVID-19. Press Releases 2020. https://www.gilead.com/newsand-press/press-room/press-releases/2020/6/gileadannounces-results-from-phase-3-trial-of-remdesivi r-in-patients-with-moderate-covid-19. Accessed 3 Jun 2020.

31. "Solidarity" clinical trial for COVID-19 treatments. https://www.who.int/emergencies/diseases/novelcoronavirus-2019/global-research-on-novel-corona virus-2019-ncov/solidarity-clinical-trial-for-covid19-treatments. Accessed 27 May 2020.

32. Identifier: NCT04315948, Trial of Treatments for COVID-19 in Hospitalized Adults (DisCoVeRy). 2020. https://clinicaltrials.gov/ct2/show/NCT0431 5948 ?term $=$ remdesivir\&cntry $=F R \& d r a w=2 \&$ rank $=$ 8. Accessed 31 May 2020.

33. Identifier: NCT04401579, Adaptive COVID-19 Treatment Trial 2 (ACTT-II). 2020. https:// clinicaltrials.gov/ct2/show/NCT04401579. Accessed 17 June 2020. 